\title{
Insufficient sleep duration is associated with worse self-rated health and more psychosomatic health complaints in adolescents
}

\author{
Kosticova $\mathrm{M}^{1}$, Madarasova Geckova $\mathrm{A}^{2}$, Dobiasova $\mathrm{E}^{1}$, Dankulincova Veselska $\mathrm{Z}^{2}$ \\ Institute of Social Medicine and Medical Ethics, Faculty of Medicine, Comenius University in Bratislava, \\ Bratislava, Slovakia.michaela.kosticova@fmed.uniba.sk
}

\section{ABSTRACT}

OBJECTIVE: To investigate the association between sleep duration and self-rated health and psychosomatic health complaints in a representative sample of Slovak adolescents aged 13 and 15 years.

BACKGROUND: According to empirically supported recommendations adolescents age 13 to 18 years old should sleep 8 to 10 hours per 24 hours on a regular basis to promote optimal health.

METHODS: We used data from the Health Behaviour in School-aged Children (HBSC) study conducted in 2018 in Slovakia comprising 8,405 adolescents aged from 11 to 15 years (mean age 13.43; 50.9\% boys). Presented are results for specific age groups of 13-years old $(n=1,909)$ and 15-years old $(n=1,293)$ adolescents. Linear regression models adjusted for age and gender were used to asses associations between sleep duration and self-rated health and psychosomatic complaints. The modifications of the associations by age and gender were also explored.

RESULTS: Insufficient sleep duration (less than 8hours) was significantly associated with worse self-rated health and more overall as well as specific psychosomatic health complaints including headache, backache, feeling low, irritability and bad temper, feeling nervous, sleeping difficulties and feeling dizzy.

CONCLUSION: In order to promote the health and well-being of adolescents, interventions to improve sleep duration are needed (Tab. 3, Ref. 36). Text in PDF www.elis.sk. KEY WORDS: insufficient sleep, adolescents, subjective health, health complaints.

\section{Introduction}

Sleep is fundamental to children's health and well-being. According to the recommendations of the American Academy of Sleep Medicine adolescents 13 to 18 years old should sleep 8 to 10 hours per 24 hours on a regular basis to promote optimal health $(1,2)$. Sleeping for the recommended number of hours on a regular basis is associated with better physical and mental health outcomes, including improved cognitive performance, attention, behavior, emotional regulation and quality of life (3-5).

Recent studies indicate that sleep duration is consistently decreasing over time and across adolescence and adolescents today are not getting enough sleep (6-9). Over the last 100 years there has been more than 1-hour decline of children's and adolescents' sleep duration as a result of progressive delays in bedtimes, but unchanged wake times (6). As adolescents get older, they prefer

${ }^{1}$ Institute of Social Medicine and Medical Ethics, Faculty of Medicine, Comenius University in Bratislava, Bratislava, Slovakia, and ${ }^{2}$ Department of Health Psychology, Faculty of Medicine, PJ Safarik University in Kosice, Kosice, Slovakia

Address for correspondence: M. Kosticova, MD, PhD, MPH, Institute of Social Medicine and Medical Ethics, Faculty of Medicine, Comenius University in Bratislava, Sasinkova 2, SK-813 72 Bratislava, Slovakia. Phone: +421.2.9119377

Acknowledgements: This work was supported by the Slovak Research and Development Agency under the Contracts No. APVV-18-0070 and No. APVV-15-0012. to go to bed later, which creates a significant sleep debt mainly during the school days $(7,9,10)$. Various factors have been shown to be associated with the late bedtimes and insufficient sleep duration in adolescence, such as shifts in the circadian rhythm during adolescence, early school start times, electronic media use during the bedtime, lack of bedtime monitoring, stress at home and school (11-15).

Evidence suggests, that insufficient sleep has significant adverse consequences on adolescent physical and mental health, cognitive functioning, emotions, behavior and safety; with increased risk of poorly perceived health, diabetes, obesity, hypertension, anxiety, depression, suicidality, substance use, behavioral problems, accidents and injuries $(4,11,12,16-18)$.

There is evidence from the studies from other countries that insufficient sleep duration is associated with worse self-rated health and more psychosomatic complaints in adolescents (19-21), but no study has been done yet in Slovakia. Such information is important to find out if Slovak adolescents are also sleep deprived and how sleep deprivation is affecting their health in order to identify the need for interventions to improve the sleep duration in Slovak adolescents.

The aim of this study was to investigate the association between sleep duration and self-rated health and psychosomatic health complaints in a representative sample of Slovak adolescents age 13 and 15 years. Empirically supported sleep recommendations will be used to operationalize total sleep time, which recommends $8-10$ hours of sleep per night on a regular basis for 
13 to 18 years old adolescents (2). Our study aimed to find out if Slovak adolescents, who slept less than recommended, perceived their health worse and suffered from more health complaints than adolescents with sufficient sleep duration.

\section{Methods}

\section{Sample and procedure}

We used data from the Health Behaviour in School-aged Children (HBSC) study conducted in 2018 in Slovakia. The HBSC used a two-step sampling to obtain a representative sample. In the first step, 140 larger and smaller elementary schools located in rural as well as in urban areas from all regions in Slovakia were asked to participate. These were randomly selected from a list of all eligible schools in Slovakia obtained from the Slovak Institute of Information and Prognosis for Education. The school response rate (RR) was $77.9 \%$. In the second step, we obtained data from 8,405 adolescents from the fifth to ninth grades of the elementary schools in Slovakia in the target group of 11 to 15 years old (mean age $13.43 ; 50.9 \%$ boys); one class per grade was selected. Presented are results for specific age groups of 13- and 15-years old adolescents.

\section{Ethics}

The study was approved by the Ethics Committee of the Medical Faculty at the P.J. Safarik University in Kosice (16N/2017). Parents were informed about the study via the school administration and could opt out if they disagreed with their child's participa- tion. Participation in the study was fully voluntary and anonymous with no explicit incentives provided for participation.

\section{Measures}

Sleep duration during school days was calculated by estimating the time between bedtime and get up time. Bedtime was measured with the item: "At what time do you usually go to bed, when you are going to school the morning after?" Get up time was measured with the item: "At what time do you usually get up, when you are going to school?" According to the recommendations of the American Academy of Sleep Medicine (2) 13- and 15-years old adolescents should sleep 8-10 hours regularly. Sleep durations less than 8 hours were coded as "insufficient", sleep durations 8 hours and more as "sufficient".

Self-rated health was measured using the single item "Would you say your health is...?" with the response categories "excellent", "good", "fair", and "poor", and response categories where dichotomized ("excellent" and "good" vs. "fair", and "poor") as recommended by the HBSC study methodology (22).

The HBSC-symptoms checklist (HBSC-SCL) assessed the occurrence of eight subjective psychosomatic health complaints: headache, stomachache, backache, feeling low, irritability and bad temper, feeling nervous, sleeping difficulties and feeling dizzy. The response categories indicating how frequently during the last 6 months the symptoms had occurred are "rarely or never", "about every month", "about every week", "more than once a week" and "about every day". Responses for specific health complaints were dichotomized ("rarely or never" and "about every monthô vs

Tab. 1. Descriptive statistics of studied variables stratified by age and gender (Slovakia, 2018, 13- and 15-years old).

\begin{tabular}{|c|c|c|c|c|}
\hline & \multicolumn{2}{|c|}{$\begin{array}{c}13 \text {-years old } \\
\mathrm{n}=1,909\end{array}$} & \multicolumn{2}{|c|}{$\begin{array}{c}15 \text {-years old } \\
\mathrm{n}=1,293\end{array}$} \\
\hline & $\begin{array}{l}\text { boys } \\
\mathrm{n}, \%\end{array}$ & $\begin{array}{l}\text { girls } \\
\mathrm{n}, \%\end{array}$ & $\begin{array}{l}\text { boys } \\
\mathrm{n}, \%\end{array}$ & $\begin{array}{l}\text { girls } \\
\mathrm{n}, \%\end{array}$ \\
\hline $\begin{array}{l}\text { Sleep duration (N, \%) } \\
\text { sufficient sleep duration ( } 8 \text { hours and more) }\end{array}$ & $675,70.2 \%$ & $597,65.5 \%$ & $363,53.1 \%$ & $289,49.7 \%$ \\
\hline $\begin{array}{l}\text { Self-rated health }(\mathbf{N}, \mathbf{\%}) \\
\text { fair and poor }\end{array}$ & $111,11.3 \%$ & $137,14.8 \%$ & $80,11.4 \%$ & $100,17.0 \%$ \\
\hline $\begin{array}{l}\text { Health complaints (N, \%) } \\
2 \text { and more at least once a week }\end{array}$ & $303,35.7 \%$ & $364,46.8 \%$ & $216,35.8 \%$ & $273,52.8 \%$ \\
\hline $\begin{array}{l}\text { Headache }(\mathbf{N}, \%) \\
\text { at least once a week }\end{array}$ & $109,11.4 \%$ & $173,19.2 \%$ & $76,11.2 \%$ & $146,25.4 \%$ \\
\hline $\begin{array}{l}\text { Stomach ache }(\mathbf{N}, \%) \\
\text { at least once a week }\end{array}$ & $87,9.4 \%$ & $127,14.4 \%$ & $45,6.8 \%$ & $73,13.0 \%$ \\
\hline $\begin{array}{l}\text { Backache (N, \%) } \\
\text { at least once a week }\end{array}$ & $140,15.1 \%$ & $156,17.9 \%$ & $111,16.6 \%$ & $117,20.7 \%$ \\
\hline $\begin{array}{l}\text { Feeling low }(\mathbf{N}, \%) \\
\text { at least once a week }\end{array}$ & $83,9.3 \%$ & $170,20.1 \%$ & $63,9.7 \%$ & $113,20.6 \%$ \\
\hline $\begin{array}{l}\text { Irritability }(\mathbf{N}, \mathbf{\%}) \\
\text { at least once a week }\end{array}$ & $279,30.3 \%$ & $329,37.6 \%$ & $226,34.1 \%$ & $236,42.1 \%$ \\
\hline $\begin{array}{l}\text { Feeling nervous (N, \%) } \\
\text { at least once a week }\end{array}$ & $324,35.3 \%$ & $343,39.8 \%$ & $195,29.5 \%$ & $237,42.9 \%$ \\
\hline $\begin{array}{l}\text { Sleeping difficulties (N, \%) } \\
\text { at least once a week }\end{array}$ & $158,17.1 \%$ & $234,27.1 \%$ & $111,17.0 \%$ & $173,31.2 \%$ \\
\hline $\begin{array}{l}\text { Feeling dizzy }(\mathbf{N}, \%) \\
\text { at least once a week }\end{array}$ & $65,7.1 \%$ & $144,16.5 \%$ & $63,9.6 \%$ & $104,18.5 \%$ \\
\hline
\end{tabular}


"about every week", "more than once a week" and "about every day"). Also, recurrent multiple health complaints were computed and subsequently dichotomized, with two or more complaints at least once a week considered as representing noticeable subjective health complaints (23).

\section{Statistical analysis}

First, we described the sample using descriptive statistics. Second, we assessed the associations of the sleep duration with self-rated health and psychosomatic health complaints using univariate logistic regression analysis crude and adjusted for age and gender. Finally, we explored the modification of the associations of sleep duration with self-rated health and psychosomatic health complaints by age and gender. Statistical analyses were performed using SPSS v.20.

\section{Results}

Table 1 shows the basic descriptive statistics of the studied variables in the whole sample as well as stratified by age and gender.

Tables 2 and 3 present the associations from univariate logistic regression models of sleep duration with self-rated health and psychosomatic health complaints, adjusted for age and gender. More girls than boys rated their health worse and suffered from overall as well as specific psychosomatic complaints. Sleep duration was significantly associated with self-rated health and psychosomatic health complaints, with insufficient sleep duration increasing the probability of worse self-rated health, overall psychosomatic health complaints as well as specific psychosomatic health complaints (headache, backache, feeling low, irritability and bad temper, feeling nervous, sleeping difficulties and feeling dizzy). Interactions of sleep duration with age and gender were tested but were not found to be significant. The only exception was the interaction of sleep duration and gender with headache. Insufficient sleep duration among girls was associated with a higher probability of headaches at least once a week.

\section{Discussion}

The present study in a representative sample of Slovak adolescents demonstrates the association between sleep duration and self-rated health and health complaints. Half of the 15 -years old and one third of 13-years old adolescents slept less than recommended 8 hours during the school days. Insufficient sleep duration was associated with worse self-rated health and more overall as well as specific psychosomatic health complaints including headache, backache, feeling low, irritability and bad temper, feeling nervous, sleeping difficulties and feeling dizzy. Health complaints were more common in girls than in boys, no gender differences were found in the frequency of health complaints in sleep deprived adolescents, except headaches, which were more common in sleep deprived girls.

Our results are in line with the results of the Health Behaviour in School-aged Children (HBSC) studies conducted in other countries $(19,21,24)$ as well as other studies worldwide $(18,20)$. The studies from Sweden (21) and Spain (20) confirmed our findings, that adolescents aged 11- to 15-years who slept less than recommended, had higher odds of all specific complaints and they also found, that perceived sleep quality had a greater impact on perceived health than total sleep time. Although sleep duration is a necessary component of "optimal sleep," sleep quality also plays an important role and is a strong predictor of physical and mental health $(5,25)$. In our study, $27.1 \%$ of 13 -years old and $31.2 \%$ of 15 -years old girls reported sleeping difficulties once a week, and adolescents who slept less than recommended reported sleeping difficulties more often. Evidence from HBSC study in Canadian adolescents showed that not just sleep duration, but also the timing

Tab. 2. The association of sleep duration with self-rated health and psychosomatic health complaints and the moderation by age and gender from univariate logistic regression (Odds ratios - OR, 95\% Confidence intervals - 95\% CI) (Slovakia, 2018, 13- and 15-years old).

\begin{tabular}{|c|c|c|c|c|c|c|}
\hline & \multicolumn{3}{|c|}{$\begin{array}{l}\text { Self-rated health } \\
\text { (fair and poor) }\end{array}$} & \multicolumn{3}{|c|}{$\begin{array}{c}\text { Health complaints } \\
\text { (2 and more at least once a week) }\end{array}$} \\
\hline & $\begin{array}{c}\text { Model } 1 \\
\text { OR, } 95 \% \text { CI }\end{array}$ & $\begin{array}{c}\text { Model } 2 \\
\text { OR, } 95 \% \text { CI }\end{array}$ & $\begin{array}{c}\text { Model } 3 \\
\text { OR, } 95 \% \text { CI }\end{array}$ & $\begin{array}{c}\text { Model } 1 \\
\text { OR, } 95 \% \mathrm{CI}\end{array}$ & $\begin{array}{c}\text { Model } 2 \\
\text { OR, } 95 \% \text { CI }\end{array}$ & $\begin{array}{c}\text { Model } 3 \\
\text { OR, } 95 \% \text { CI }\end{array}$ \\
\hline $\begin{array}{l}\text { Gender } \\
\text { boys } \\
\text { girls } \\
\end{array}$ & $\begin{array}{l}\text { Ref. } \\
1.431 .16 \mid 1.76^{* * *} \\
\end{array}$ & $\begin{array}{c}\text { Ref. } \\
1.300 .99 \mid 1.71 \\
\end{array}$ & $\begin{array}{c}\text { Ref. } \\
1.431 .16 \mid 1.76^{* * *}\end{array}$ & $\begin{array}{l}\text { Ref. } \\
1.731 .48 \mid 2.02^{* * *}\end{array}$ & $\begin{array}{c}\text { Ref. } \\
1.561 .28 \mid 1.91^{* * *}\end{array}$ & $\begin{array}{l}\text { Ref. } \\
1.731 .48 \mid 2.02^{* * *}\end{array}$ \\
\hline $\begin{array}{l}\text { Age } \\
13 \text {-years old } \\
15 \text {-years old } \\
\end{array}$ & $\begin{array}{c}\quad \text { Ref. } \\
1.020 .83 \mid 1.27\end{array}$ & $\begin{array}{cl} & \text { Ref. } \\
1.03 & 0.83 \mid 1.27 \\
\end{array}$ & $\begin{array}{c}\text { Ref. } \\
1.200 .90 \mid 1.60 \\
\end{array}$ & $\begin{array}{cl} & \text { Ref. } \\
1.03 & 0.88 \mid 1.21 \\
\end{array}$ & $\begin{array}{ll} & \text { Ref. } \\
1.03 & 0.88 \mid 1.21 \\
\end{array}$ & $\begin{array}{c}\quad \text { Ref. } \\
1.040 .85 \mid 1.29 \\
\end{array}$ \\
\hline $\begin{array}{l}\text { Sleep duration } \\
\text { sufficient (8hours and more) } \\
\text { insufficient (less than } 8 \text { hours) }\end{array}$ & $\begin{array}{c}\text { Ref. } \\
1.361 .10 \mid 1.68^{* *} \\
\end{array}$ & $\begin{array}{c}\text { Ref. } \\
1.210 .88 \mid 1.65 \\
\end{array}$ & $\begin{array}{c}\text { Ref. } \\
1.571 .19 \mid 2.07^{* * *} \\
\end{array}$ & $\begin{array}{c}\text { Ref. } \\
1.711 .46 \mid 2.00^{* * * *}\end{array}$ & $\begin{array}{c}\text { Ref. } \\
1.511 .20 \mid 1.88^{* * *} \\
\end{array}$ & $\begin{array}{c}\text { Ref. } \\
1.721 .39 \mid 2.13^{* * *} \\
\end{array}$ \\
\hline $\begin{array}{l}\text { Sleep duration*Gender } \\
\text { sufficient*boys } \\
\text { insufficient*girls }\end{array}$ & & $\begin{array}{c}\text { Ref. } \\
1.240 .82 \mid 1.89 \\
\end{array}$ & & & $\begin{array}{cl} & \text { Ref. } \\
1.29 & 0.94 \mid 1.77 \\
\end{array}$ & \\
\hline $\begin{array}{l}\text { Sleep duration*Age } \\
\text { sufficient*13-years old } \\
\text { insufficient*15-years old }\end{array}$ & & & $\begin{array}{l}\text { Ref. } \\
0.710 .47 \mid 1.09\end{array}$ & & & $\begin{array}{rl} & \text { Ref. } \\
0.98 & 0.71 \mid 1.35\end{array}$ \\
\hline
\end{tabular}


Tab. 3. The association of sleep duration with specific psychosomatic health complaints and the moderation by age and gender from univariate logistic regression (Odds ratios - OR, 95\% Confidence intervals - 95\%CI) (Slovakia, 2018, 13- and 15-years old).

\begin{tabular}{|c|c|c|c|c|c|c|}
\hline & \multicolumn{3}{|c|}{ Headache } & \multicolumn{3}{|c|}{ Stomachache } \\
\hline & $\begin{array}{c}\text { Model } 1 \\
\text { OR, 95\%CI }\end{array}$ & $\begin{array}{c}\text { Model } 2 \\
\text { OR, 95\%CI }\end{array}$ & $\begin{array}{c}\text { Model } 3 \\
\text { OR, 95\%CI }\end{array}$ & $\begin{array}{c}\text { Model } 1 \\
\text { OR, 95\%CI }\end{array}$ & $\begin{array}{c}\text { Model } 2 \\
\text { OR, 95\%CI }\end{array}$ & $\begin{array}{c}\text { Model } 3 \\
\text { OR, 95\%CI }\end{array}$ \\
\hline $\begin{array}{l}\text { Gender } \\
\text { boys } \\
\text { girls }\end{array}$ & $\begin{array}{c}\text { Ref. } \\
2.211 .81 \mid 2.70^{* * *}\end{array}$ & $\begin{array}{c}\text { Ref. } \\
1.751 .33 \mid 2.30^{* * *}\end{array}$ & $\begin{array}{c}\text { Ref. } \\
2.211 .81 \mid 2.70^{* * *}\end{array}$ & $\begin{array}{c}\text { Ref. } \\
1.781 .41 \mid 2.26^{* * *}\end{array}$ & $\begin{array}{c}\text { Ref. } \\
1.641 .20 \mid 2.23^{* *}\end{array}$ & $\begin{array}{c}\text { Ref. } \\
1.781 .41 \mid 2.26^{* * *}\end{array}$ \\
\hline $\begin{array}{l}\text { Age } \\
13 \text {-years old } \\
15 \text {-years old }\end{array}$ & $\begin{array}{c}\text { Ref. } \\
1.120 .92 \mid 1.37\end{array}$ & $\begin{array}{c}\text { Ref. } \\
1.130 .92 \mid 1.38\end{array}$ & $\begin{array}{c}\text { Ref. } \\
1.040 .78 \mid 1.39\end{array}$ & $\begin{array}{c}\text { Ref. } \\
0.770 .60 \mid 0.99^{*}\end{array}$ & $\begin{array}{c}\text { Ref. } \\
0.770 .60 \mid 0.99^{*}\end{array}$ & $\begin{array}{c}\quad \text { Ref. } \\
0.780 .56 \mid 1.09\end{array}$ \\
\hline $\begin{array}{l}\text { Sleep duration } \\
\text { sufficient ( } 8 \text { hours and more) } \\
\text { insufficient (less than } 8 \text { hours) }\end{array}$ & $\begin{array}{c}\text { Ref. } \\
1.691 .39 \mid 2.07^{* * *}\end{array}$ & $\begin{array}{c}\text { Ref. } \\
1.240 .90 \mid 1.71 \\
\end{array}$ & $\begin{array}{c}\text { Ref. } \\
1.581 .22 \mid 2.06^{* * *} \\
\end{array}$ & $\begin{array}{c}\quad \text { Ref. } \\
1.230 .97 \mid 1.56 \\
\end{array}$ & $\begin{array}{c}\text { Ref. } \\
1.090 .74 \mid 1.58 \\
\end{array}$ & $\begin{array}{c}\text { Ref. } \\
1.240 .92 \mid 1.68 \\
\end{array}$ \\
\hline $\begin{array}{l}\text { Sleep duration*Gender } \\
\text { sufficient*boys } \\
\text { insufficient*girls }\end{array}$ & & $\begin{array}{c}\text { Ref. } \\
1.651 .10 \mid 2.48^{*}\end{array}$ & & & $\begin{array}{c}\quad \text { Ref. } \\
1.230 .76 \mid 1.99\end{array}$ & \\
\hline $\begin{array}{l}\text { Sleep duration*Age } \\
\text { sufficient*13-years old } \\
\text { insufficient* } 15 \text {-years old }\end{array}$ & & & $\begin{array}{c}\text { Ref. } \\
1.170 .78 \mid 1.75 \\
\end{array}$ & & & $\begin{array}{c}\text { Ref. } \\
0.970 .59 \mid 1.59 \\
\end{array}$ \\
\hline $\begin{array}{l}\text { Gender } \\
\text { boys } \\
\text { girls }\end{array}$ & $\begin{array}{c}\text { Ref. } \\
1.221 .01 \mid 1.48^{*}\end{array}$ & $\begin{array}{c}\text { Ref. } \\
1.220 .93 \mid 1.60\end{array}$ & $\begin{array}{c}\text { Ref. } \\
1.221 .01 \mid 1.48^{*}\end{array}$ & $\begin{array}{c}\text { Ref. } \\
2.411 .94 \mid 2.99^{* * *}\end{array}$ & $\begin{array}{c}\text { Ref. } \\
2.311 .72 \mid 3.10^{* * *}\end{array}$ & $\begin{array}{c}\text { Ref. } \\
2.411 .94 \mid 2.99^{* * *}\end{array}$ \\
\hline $\begin{array}{l}\text { Age } \\
13 \text {-years old } \\
15 \text {-years old }\end{array}$ & $\begin{array}{c}\quad \text { Ref. } \\
1.030 .85 \mid 1.26\end{array}$ & $\begin{array}{c}\quad \text { Ref. } \\
1.030 .85 \mid 1.26\end{array}$ & $\begin{array}{c}\text { Ref. } \\
1.280 .97 \mid 1.69\end{array}$ & $\begin{array}{c}\quad \text { Ref. } \\
0.950 .76 \mid 1.18\end{array}$ & $\begin{array}{c}\quad \text { Ref. } \\
0.950 .76 \mid 1.18\end{array}$ & $\begin{array}{c}\text { Ref. } \\
0.940 .70 \mid 1.28\end{array}$ \\
\hline $\begin{array}{l}\text { Sleep duration } \\
\text { sufficient ( } 8 \text { hours and more) } \\
\text { insufficient (less than } 8 \text { hours) }\end{array}$ & $\begin{array}{c}\text { Ref. } \\
1.851 .53 \mid 2.25^{* * *}\end{array}$ & $\begin{array}{c}\text { Ref. } \\
1.851 .40 \mid 2.44^{* * *}\end{array}$ & $\begin{array}{c}\text { Ref. } \\
2.231 .73 \mid 2.88^{* * *}\end{array}$ & $\begin{array}{c}\text { Ref. } \\
1.561 .26 \mid 1.94^{* * *}\end{array}$ & $\begin{array}{c}\text { Ref. } \\
1.481 .04 \mid 2.10^{*}\end{array}$ & $\begin{array}{c}\text { Ref. } \\
1.561 .18 \mid 2.06^{* *}\end{array}$ \\
\hline $\begin{array}{l}\text { Sleep duration*Gender } \\
\text { sufficient*boys } \\
\text { insufficient*girls }\end{array}$ & & $\begin{array}{c}\text { Ref. } \\
1.010 .69 \mid 1.48\end{array}$ & & & & $\begin{array}{c}\quad \text { Ref. } \\
1.090 .71 \mid 1.69\end{array}$ \\
\hline $\begin{array}{l}\text { Sleep duration*Age } \\
\text { sufficient*13-years old } \\
\text { insufficient* } 15 \text {-years old }\end{array}$ & & & $\begin{array}{c}\text { Ref. } \\
0.650 .44 \mid 1.00\end{array}$ & & & $\begin{array}{c}\quad \text { Ref. } \\
1.010 .65 \mid 1.56\end{array}$ \\
\hline $\begin{array}{l}\text { Gender } \\
\text { boys } \\
\text { girls }\end{array}$ & $\begin{array}{c}\text { Ref. } \\
1.381 .19 \mid 1.61^{* * *}\end{array}$ & $\begin{array}{c}\text { Ref. } \\
1.271 .04 \mid 1.55^{*}\end{array}$ & $\begin{array}{c}\text { Ref. } \\
1.381 .18 \mid 1.60^{* * *}\end{array}$ & $\begin{array}{c}\text { Ref. } \\
1.411 .21 \mid 1.63^{* * *}\end{array}$ & $\begin{array}{c}\text { Ref. } \\
1.421 .16 \mid 1.72^{* * *}\end{array}$ & $\begin{array}{c}\text { Ref. } \\
1.401 .21 \mid 1.63^{* * *}\end{array}$ \\
\hline $\begin{array}{l}\text { Age } \\
13 \text {-years old } \\
15 \text {-years old }\end{array}$ & $\begin{array}{c}\text { Ref. } \\
1.120 .95 \mid 1.30\end{array}$ & $\begin{array}{l}\text { Ref. } \\
1.120 .95 \mid 1.31\end{array}$ & $\begin{array}{c}\text { Ref. } \\
1.180 .96 \mid 1.46\end{array}$ & $\begin{array}{c}\quad \text { Ref. } \\
0.890 .76 \mid 1.04\end{array}$ & $\begin{array}{c}\text { Ref. } \\
0.890 .76 \mid 1.04\end{array}$ & \begin{tabular}{c}
\multicolumn{1}{c}{ Ref. } \\
$0.990 .80 \mid 1.21$
\end{tabular} \\
\hline $\begin{array}{l}\text { Sleep duration } \\
\text { sufficient ( } 8 \text { hours and more) } \\
\text { insufficient (less than } 8 \text { hours) }\end{array}$ & $\begin{array}{c}\text { Ref. } \\
1.551 .33 \mid 1.81^{* * *}\end{array}$ & $\begin{array}{c}\text { Ref. } \\
1.411 .13 \mid 1.76^{* *}\end{array}$ & $\begin{array}{c}\text { Ref. } \\
1.641 .34 \mid 2.02^{* * *}\end{array}$ & $\begin{array}{c}\text { Ref. } \\
1.441 .23 \mid 1.68^{* * *}\end{array}$ & $\begin{array}{c}\text { Ref. } \\
1.451 .17 \mid 1.81^{* * *}\end{array}$ & $\begin{array}{c}\text { Ref. } \\
1.591 .29 \mid 1.95^{* * *}\end{array}$ \\
\hline $\begin{array}{l}\text { Sleep duration*Gender } \\
\text { sufficient*boys } \\
\text { insufficient*girls }\end{array}$ & & 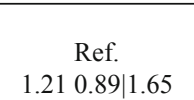 & & & $\begin{array}{ll} & \text { Ref. } \\
0.98 & 0.72 \mid 1.33\end{array}$ & \\
\hline $\begin{array}{l}\text { Sleep duration*Age } \\
\text { sufficient*13-years old } \\
\text { insufficient* } 15 \text {-years old }\end{array}$ & & & $\begin{array}{c}\text { Ref. } \\
0.880 .64 \mid 1.20\end{array}$ & & & $\begin{array}{cl} & \text { Ref. } \\
0.79 & 0.58 \mid 1.09 \\
\end{array}$ \\
\hline $\begin{array}{l}\text { Gender } \\
\text { boys } \\
\text { girls }\end{array}$ & $\begin{array}{c}\text { Ref. } \\
1.931 .62 \mid 2.31^{* * *}\end{array}$ & $\begin{array}{c}\text { Ref. } \\
1.821 .42 \mid 2.33^{* * *}\end{array}$ & $\begin{array}{c}\text { Ref. } \\
1.931 .62 \mid 2.31^{* * *}\end{array}$ & $\begin{array}{c}\text { Ref. } \\
2.351 .87 \mid 2.96^{* * *}\end{array}$ & $\begin{array}{c}\text { Ref. } \\
2.241 .62 \mid 3.08^{* * *}\end{array}$ & $\begin{array}{c}\text { Ref. } \\
2.351 .87 \mid 2.96^{* * *}\end{array}$ \\
\hline $\begin{array}{l}\text { Age } \\
13 \text {-years old } \\
15 \text {-years old }\end{array}$ & \begin{tabular}{c}
\multicolumn{1}{c}{ Ref. } \\
$0.970 .81 \mid 1.17$
\end{tabular} & \begin{tabular}{c}
\multicolumn{1}{c}{ Ref. } \\
$0.970 .81 \mid 1.17$
\end{tabular} & $\begin{array}{c}\text { Ref. } \\
0.940 .72 \mid 1.23\end{array}$ & 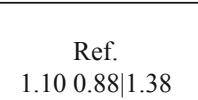 & $\begin{array}{l}\text { Ref. } \\
1.100 .88 \mid 1.38\end{array}$ & $\begin{array}{c}\quad \text { Ref. } \\
1.240 .90 \mid 1.72\end{array}$ \\
\hline $\begin{array}{l}\text { Sleep duration } \\
\text { sufficient ( } 8 \text { hours and more) } \\
\text { insufficient (less than } 8 \text { hours) }\end{array}$ & $\begin{array}{c}\text { Ref. } \\
2.081 .74 \mid 2.49^{* * *}\end{array}$ & $\begin{array}{c}\text { Ref. } \\
1.941 .48 \mid 2.54^{* * *}\end{array}$ & $\begin{array}{c}\text { Ref. } \\
2.031 .61 \mid 2.57^{* * *}\end{array}$ & $\begin{array}{c}\text { Ref. } \\
1.721 .38 \mid 2.16^{* * *}\end{array}$ & $\begin{array}{l}\text { Ref. } \\
1.611 .11 \mid 2.34^{*}\end{array}$ & $\begin{array}{c}\text { Ref. } \\
1.901 .42 \mid 2.56^{* * *}\end{array}$ \\
\hline $\begin{array}{l}\text { Sleep duration*Gender } \\
\text { sufficient*boys } \\
\text { insufficient*girls }\end{array}$ & & $\begin{array}{c}\text { Ref. } \\
1.140 .80 \mid 1.62\end{array}$ & & & \begin{tabular}{ll}
\multicolumn{1}{c}{ Ref. } \\
1.11 & $0.70 \mid 1.76$
\end{tabular} & \\
\hline $\begin{array}{l}\text { Sleep duration*Age } \\
\text { sufficient*13-years old } \\
\text { insufficient*15-years old }\end{array}$ & & & $\begin{array}{c}\text { Ref. } \\
1.060 .74 \mid 1.53\end{array}$ & & & $\begin{array}{cl} & \text { Ref. } \\
0.79 & 0.51 \mid 1.25\end{array}$ \\
\hline
\end{tabular}


of sleep is related to adolescent health. Adolescents who went to sleep later, independently on sleep duration, reported worse health and more health complaints (26). The findings from our previous study in the same sample of Slovak adolescents suggested that when adolescents got older, they went to sleep later (27). To our knowledge this is the first study assessing the impact of sleep deprivation on the health of adolescents in Slovakia.

The findings of our study confirm the hypothesis that sleep deprived adolescents suffer from worse self-rated health and more psychosomatic complaints. As the findings cannot demonstrate a causal relationship, questions about the underlying mechanism arise. Evidence shows that the causes and consequences of insufficient sleep duration are often closely interrelated in complex ways $(3,11)$. It is currently considered that the association between sleep duration and health complaints is likely bidirectional, negative emotions and pain may have a negative impact on sleep, and a sleep deficiency may have a negative effect on mood and health status the day after $(16,21,28)$. According to a recent systematic review, a strong causal relationship exists between sleep loss in adolescents and adverse health outcomes, however, this link may be weakened by competing influences such as emotional status and psychosocial functioning (16). Adolescence is a period during which major biological, social, physiological, and cognitive changes take place (29). Subjective health complaints, which are common in adolescents, are often viewed as a reaction to psychosocial stress and mostly based on sensations from physiological processes $(19,20)$. Evidence from experimental studies has shown that insufficient sleep causes an imbalance in physiological processes and neurohormonal changes which might increase the vulnerability of adolescents to different types of stressors and result in psychosomatic health complaints $(30,31)$ and similar mechanism might be expected based on the revealed connection between insufficient sleep and psychosomatic health problems as a part of findings in our study.

\section{Strengths and limitations}

The strengths of the study include a large representative sample, the use of valid HBSC questionnaire and empirically based sleep recommendations. Moreover, the national HBSC study is a part of the international HBSC study running in 44 countries every 4 years, so it enables to compare the data between the countries and analyse time trends.

There are some limitations of the study. First, the cross-sectional design does not allow to establish causal relationships. We are uncertain whether the insufficient sleep is a determinant or outcome of health complaints. Second, we used subjective measures of sleep duration, which are not able to measure sleep as precisely as objective measures, such as actigraphy. Nevertheless, this is common in questionnaire studies that cover a broad range of topics and a large sample of participants, such as the HBSC study. Third, the sleep duration was calculated as a difference between wake-up time and bedtime, which is the time spent in bed and not the time spent asleep. This could have caused the number of sufficient sleepers was overestimated. Finally, regarding the optimal time of sleep, we referred to sleep recommendations of the American Academy of Sleep Medicine (1), which recommends at least 8 hours of regular sleep for 13-years old adolescents, compared to 9 hours recommended by National Sleep Foundation (3). If we have used more strict recommendations of NSF, we would have a higher proportion of insufficient sleepers in 13 years old adolescents.

\section{Implications}

The results of our study suggest that health professionals and caregivers should pay attention to adolescent sleep duration since insufficient sleep is a common problem and might explain high levels of health complaints in this population. In order to promote the health and well-being of adolescents, interventions to improve sleep duration are needed. There is strong evidence that delaying school start time can be the most effective method to benefit adolescent sleep (32-36). The role of parental bedtime monitoring in relation to sleep duration is also hugely discussed in recent studies $(13,15,24)$, therefore future research is needed to investigate this association in Slovak adolescents. Future research should also focus on factors associated with insufficient sleep duration in adolescence in order to find out why adolescents go sleep late during the school nights, what they do during their time awake and how these activities influence their health.

\section{Conclusion}

In conclusion, the present study highlights the association between sleep duration and subjective health and health complaints in adolescents from Slovakia. Our findings suggest that a high proportion of Slovak adolescents is sleeping less than recommended during the school days and insufficient sleep duration is associated with worse self-rated health and more psychosomatic health complaints. Therefore, in order to promote the health and well-being of adolescents, interventions to improve sleep duration are needed.

\section{References}

1. Paruthi S, Brooks LJ, D’Ambrosio C, Hall WA, Kotagal S, Lloyd RM et al. Consensus Statement of the American Academy of Sleep Medicine on the Recommended Amount of Sleep for Healthy Children: Methodology and Discussion. J Clin Sleep Med 2016; 12 (11): 1549-1561.

2. Paruthi S, Brooks LJ, D'Ambrosio C, Hall WA, Kotagal S, Lloyd RM et al. Recommended Amount of Sleep for Pediatric Populations: A Consensus Statement of the American Academy of Sleep Medicine. J Clin Sleep Med 2016; 12 (06): 785-786.

3. Hirshkowitz M, Whiton K, Albert SM, Alessi C, Bruni O, DonCarlos L et al. National Sleep Foundation's updated sleep duration recommendations: final report. Sleep Health 2015; 1 (4): 233-243.

4. Chaput JP, Gray CE, Poitras VJ, Carson V, Gruber R, Olds T et al. Systematic review of the relationships between sleep duration and health indicators in school-aged children and youth. Appl Physiol Nutrit Metab 2016; 41 (6 (Suppl. 3)): S266-282.

5. Bruni O, Brambilla P. Impact of different recommendations on adequacy rate for sleep duration in children. Ital J Pediatr [Internet] 2017 Jan 25 [cited 2019 Jan 10]; 43. Available from: https://www.ncbi.nlm.nih. gov/pmc/articles/PMC5347816/ 
6. Matricciani L, Olds T, Petkov J. In search of lost sleep: Secular trends in the sleep time of school-aged children and adolescents. Sleep Med Rev 2012; 16 (3): 203-211.

7. Keyes KM, Maslowsky J, Hamilton A, Schulenberg J. The Great Sleep Recession: Changes in Sleep Duration Among US Adolescents, 1991-2012. Pediatrics 2015; 135 (3): 460-468.

8. Norell-Clarke A, Hagquist C. Changes in sleep habits between 1985 and 2013 among children and adolescents in Sweden. Scand J Publ Health 2017; 45 (8): 869.

9. Leger D, Beck F, Richard J-B, Godeau E. Total Sleep Time Severely Drops during Adolescence. PLOS ONE 2012; 7 (10): e45204.

10. Ojio Y, Nishida A, Shimodera S, Togo F, Sasaki T. Sleep Duration Associated with the Lowest Risk of Depression/Anxiety in Adolescents. Sleep 2016; 39 (8): 1555-1562.

11. Owens J. Insufficient Sleep in Adolescents and Young Adults: An Update on Causes and Consequences. Pediatrics 2014; 134 (3): e921-932.

12. Bauducco SV, Flink IK, Jansson-Fröjmark M, Linton SJ. Sleep duration and patterns in adolescents: correlates and the role of daily stressors. Sleep Health 2016; 2 (3): 211-218.

13. Bartel KA, Gradisar M, Williamson P. Protective and risk factors for adolescent sleep: A meta-analytic review. Sleep Med Rev 2015; 21: 72-85.

14. LeBourgeois MK, Hale L, Chang A-M, Akacem LD, Montgomery-Downs HE, Buxton OM. Digital Media and Sleep in Childhood and Adolescence. Pediatrics 2017; 140 (Suppl 2): S92-96.

15. Short MA, Kuula L, Gradisar M, Pesonen A-K. How Internal and External Cues for Bedtime Affect Sleep and Adaptive Functioning in Adolescents. Sleep Medicine [Internet] 2018 Dec [cited 2019 Jan 10]; Available from: https://linkinghub.elsevier.com/retrieve/pii/ S138994571830827X

16. Shochat T, Cohen-Zion M, Tzischinsky O. Functional consequences of inadequate sleep in adolescents: A systematic review. Sleep Med Rev 2014; 18 (1): 75-87.

17. Sarchiapone M, Mandelli L, Carli V, Iosue M, Wasserman C, Hadlaczky $\mathbf{G}$ et al. Hours of sleep in adolescents and its association with anxiety, emotional concerns, and suicidal ideation. Sleep Med 2014; 15 (2): $248-254$

18. Zhang J, Paksarian D, Lamers F, Hickie IB, He J, Merikangas KR. Sleep Patterns and Mental Health Correlates in US Adolescents. J Pediatrics 2017; 182: 137-143.

19. Paiva T, Gaspar T, Matos MG. Sleep deprivation in adolescents: correlations with health complaints and health-related quality of life. Sleep Med 2015; 16 (4): 521-527.

20. Segura-Jiménez V, Carbonell-Baeza A, Keating XD, Ruiz JR, Castro-Piñero J. Association of sleep patterns with psychological positive health and health complaints in children and adolescents. Quality Life Res 2015; 24 (4): 885-895

21. Norell-Clarke A, Hagquist C. Child and adolescent sleep duration recommendations in relation to psychological and somatic complaints based on data between 1985 and 2013 from 11 to 15 year-olds. J Adolescence 2018; 68: 12-21.
22. Currie C, Gabhainn SN, Godeau E, Roberts C, Smith R, Currie D et al. Inequalities in young people's health: HBSC international report from the 2005/2006 survey. Copenhagen: WHO; 2008206 p. (Health policy for children and adolescents).

23. Ravens-Sieberer U, Torsheim T, Hetland J, Vollebergh W, Cavallo F, Jericek $\mathbf{H}$ et al. Subjective health, symptom load and quality of life of children and adolescents in Europe. Internat J Public Health 2009; 54 (S2): 151-159.

24. Nuutinen T, Roos E, Ray C, Villberg J, Välimaa R, Rasmussen M et al. Computer use, sleep duration and health symptoms: a cross-sectional study of 15-year olds in three countries. Internat J Public Health 2014; 59 (4): 619-628.

25. Ohayon M, Wickwire EM, Hirshkowitz M, Albert SM, Avidan A, Daly FJ et al. National Sleep Foundation's sleep quality recommendations: first report. Sleep Health 2017; 3 (1): 6-19.

26. Gariépy G, Doré I, Whitehead RD, Elgar FJ. More than just sleeping in: a late timing of sleep is associated with health problems and unhealthy behaviours in adolescents. Sleep Medicine [Internet] 2018 Nov [cited 2019 Jan 10]; Available from: https://linkinghub.elsevier.com/retrieve/ pii/S1389945718308955

27. Kosticova M, Dankulincova Veselska Z, Madarasova Geckova A. Polovica 15-ročných adolescentov spí menej akoby mala: výsledky HBSC štúdie Slovensko 2017/2018. In: Hudeckova H, Jakusova V, Svihrova V, Baska T, editors. Aktuálne problémy verjeného zdravotníctva vo výskume a praxi Recenzovaný zborník vedeckých a odborných prác [Internet]. Jeséniova lekarska fakulta Univerzity Komenskeho v Martine; 2019 [cited 2019 Jun 24].p. 119-27. Available from: https://www.jfmed.uniba.sk/fileadmin/ jlf/Pracoviska/ustav-verejneho-zdravotnictva/Zborniky/zbornik_2019.pdf

28. Gregory AM, Sadeh A. Sleep, emotional and behavioral difficulties in children and adolescents. Sleep Med Rev 2012; 16 (2): 129-136.

29. Babjakova J, Sekretar S (Eds). Nutrition and Food Safety in Public Health. Bratislava: Comenius University in Bratislava; 2015. 136 p.

30. Reddy R, Palmer CA, Jackson C, Farris SG, Alfano CA. Impact of sleep restriction versus idealized sleep on emotional experience, reactivity and regulation in healthy adolescents. J Sleep Res 2017; 26 (4): 516-525.

31. Baum KT, Desai A, Field J, Miller LE, Rausch J, Beebe DW. Sleep restriction worsens mood and emotion regulation in adolescents. J Child Psychol Psychiatry 2014; 55 (2): 180-190.

32. Bowers JM, Moyer A. Effects of school start time on students' sleep duration, daytime sleepiness, and attendance: a meta-analysis. Sleep Health 2017; 3 (6): 423-431.

33. Minges KE, Redeker NS. Delayed school start times and adolescent sleep: A systematic review of the experimental evidence. Sleep Med Rev 2016; 28: 86-95.

34. Busch V, Altenburg TM, Harmsen IA, Chinapaw MJ. Interventions that stimulate healthy sleep in school-aged children: a systematic literature review. Eur J Publ Health 2017; 27 (1): 53-65.

35. Gariépy G, Janssen I, Sentenac M, Elgar FJ. School start time and sleep in Canadian adolescents. J Sleep Res 2017; 26 (2): 195-201.

36. Wahlstrom KL, Berger AT, Widome R. Relationships between school start time, sleep duration, and adolescent behaviors. Sleep Health 2017; 3 (3): $216-221$. 